\title{
D-Lactat und L-Lactat im Liquor cerebrospinalis bei akuten entzündlichen Erkrankungen des Zentralnervensystems (ZNS)
}

\author{
D-Lactate and L-Lactate in Cerebrospinal Fluid with acute Inflammatory Diseases \\ of the Central Nervous System
}

T. O. Kleine

Med. Zentrum für Nervenheilkunde (Funktionsbereich Neurochemie) der Universität Marburg a. d. Lahn

\section{Zusammenfassung:}

Der Monotest für L-Lactat (Boehringer Mannheim) wurde für die Messung von D-Lactat und L-Lactat in der gleichen Probe modifiziert und an $0,01 \mathrm{ml}$ oder $0,025 \mathrm{ml}$ Liquor cerebrospinalis in zwei Ansätzen adaptiert (Nachweisgrenze $\leq 0,20 \mathrm{mmol} / \mathrm{l}$ ). Da D-Lactatkonzentrationen in Liquor und Blutplasma bakteriellen Ursprungs sind $(8,9)$, zeigen D-Lactatspiegel von $>0,20 \mathrm{mmol} / \mathrm{L} \mathrm{Li}$ quor eine akute bakterielle Entzündung im ZNS an, wenn im simultan entnommenen Blutplasma niedrigere Werte als im $\mathrm{Li}$ quor gemessen werden. Höhere Plasmawerte indizieren eine extrazerebrale Entzündung mit D-Lactat-produzierenden Bakterien. Dieser einfache Test eignet sich in der Notfallmedizin, Lactatazidosen in D-Lactat (bakteriellen Ursprungs) und L-Lactat (anaerober Metabolit) schnell zu differenzieren. Mit diesem modifizierten Monotest kann eine akute Entzündung, durch D-Lactat-produzierende Bakterien hervorgerufen, von anderen (abakteriellen) Entzündungen schnell unterschieden werden (auch nach Antibiotika-Anbehandlung); die Wirkung einer Antibiotika-Therapie ist mit diesem Test kontrollierbar.

\section{Schlüsselwörter:}

D-Lactat - L-Lactat - Liquor cerebrospinalis - Differenzierung bakterieller von abakteriellen Entzündungen

\section{Summary:}

The Monotest Lactate (Boehringer Mannheim) was modified here to measure D-lactate and L-lactate in the same sample enzymatically; it was adapted to $0.010 \mathrm{ml}$ or $0.025 \mathrm{ml}$ CSF samples displaying a detection limit of $\leq 0.20 \mathrm{mmol} / \mathrm{l}$. As D-lactate is exclusively of bacterial origin in CSF and blood $(8,9)$, concentrations $>0.20 \mathrm{mmol} / \mathrm{l}$ in CSF indicate a bacterial inflammation in CNS when D-lactate levels in the plasma sample collected simultaneously, are lower. Higher levels in blood indicate an extracerebral inflammation by D-lactate producing bacteria. The simple test is useful for the fast differentiation of lactic acidosis into D-lactate of bacterial origin and L-lactate, an anaerobical metabolite. The test rapidly discriminates an acute inflammation caused by D-lactate producing bacteria, from nonbacterial in flammations also after the begin of an antibiotic therapy; it may control therapy effect of antibiotics.

\section{Keywords:}

D-lactate - L-lactate - cerebrospinal fluid - differentiation of bacterial and nonbacterial.inflammations

\section{Einleitung}

Akute bakterielle Meningitiden können bei einem erhöhten LLactatgehalt im Liquor von $\geq 3,5 \mathrm{mmol} / \mathrm{l}$ mit einer Sensitivität von $100 \%$ und einer Spezifität von $92,7 \%$ im Lactattest erkannt werden $(1,2)$. Wird eine Leukozytenzahl von $\geq 800 / \mu$ l zur Beurteilung hinzugezogen, dann steigt die Spezifität des Lactat-plusLeukozyten-Tests auf $99,3 \%$ an (2). Nach Anbehandlung von bakteriellen Meningitiden mit Antibiotika fällt jedoch die Sensitivität des Lactattests auf 50 bis $80 \%$ (3). Enthält das zu untersuchende Patientenkollektiv nicht nur akute Meningitiden, sondern auch neurochirurgische Patienten mit z. B. akuten intrazerebralen Blutungen mit Schocksymptomatik oder Tumoren des ZNS, dann nehmen Sensitivität und Spezifität beider Lactatteste weiter ab (vgl. 3), auch bei der Anwendung anderer Grenzkonzentrationen von L-Lactat im Liquor (4-6). Bei Blutungen in die Liquorräume zeigte der Lactat-Plus-Erythrozyten-Test eine höhere Spezifität als der Lactattest allein (2). Auch eine Kombination des Lactattests mit freigesetzten Granulozytenbestandteilen im Liquor brachte Verbesserungen bei der Diagnostik akuter Meningitiden (7). Trotzdem werden nicht alle akuten Erkrankungen des ZNS mit diesen Tests erfaßt und befriedigend von anderen Krankheitszuständen differenziert.

Deshalb wurde hier ein käuflicher Lactat-Monotest für die enzymatische Messung von D-Lactat und L-Lactat in der gleichen Probe modifiziert und an Liquor adaptiert. D-Lactat wird $u$. a. von Bakterien produziert, die eine Meningitis verursachen, wie z.B. Haemophilus influenzae, Neisseria meningitidis, Escherichia coli, Streptokokken, Staphylokokken u. a. m. (vgl. 8). Da die Bildung von D-Lactat bakterienspezifisch ist und D-Lactat im Säugetierorganismus weder synthetisiert noch unter normalen $\mathrm{Be}$ dingungen verstoffwechselt wird (9), zeigt sein Nachweis im $\mathrm{Li}$ quor cerebrospinalis bzw. in anderen Körperflüssigkeiten die Anwesenheit von D-Lactat-produzierenden Bakterien an.

\section{Material und Methoden}

Liquorproben (Lumbal-, Subokzipital-(SOP-), Ventrikel-DrainageLiquor) wurden für Routineuntersuchungen simultan mit Fluorid/EDTA-Plasma gewonnen. Nach Ermittlung der Leukozytenund Erythrozytenzahl nach (2) sowie von Gesamtprotein und Glucose nach (3) wurden die Proben bei $-20^{\circ} \mathrm{C}$ bis zur Analyse gelagert.

\section{Messung von D-Lactat und L-Lactat im gleichen Test}

Der Monotest Lactat (Boehringer Mannheim $\mathrm{GmbH}$ ) wurde für die enzymatische Messung von D-Lactat und L-Lactat (vgl. 10, 11) in der gleichen Probe wie folgt modifiziert:

Ansatz $A$ in Mikroküvetten:

$0,300 \mathrm{ml}$ Reagenzlösung,

$0,010 \mathrm{ml}$ Probe (Liquor, Plasma, Kontrollen),

$0,010 \mathrm{ml}$ Enzymsuspension (L-LDH EC 1.1.1.27) plus GPT (EC 2.6.1.2),

$0,010 \mathrm{ml} \mathrm{D}-\mathrm{LDH}$-Suspension (EC 1.1.1.28).

Die Messung erfolgte in Mikroküvetten $(\mathrm{d}=1 \mathrm{~cm}$ ) im Photometer. Eppendorf mit Schreiber (Eppendorf Gerätebau, Hamburg) bei $334 \mathrm{~nm} 10 \mathrm{~min}$ bei $25^{\circ} \mathrm{C}$. Es wurden zwei Leerwerte ermittelt:

Leerwert 1 enthielt je $0,010 \mathrm{ml}\left(\mathrm{NH}_{4}\right)_{2} \mathrm{SO}_{4}$-Lösung anstatt der Enzymsuspensionen und als Probe Kontrollogen $L$ (Behringwerke Marburg);

Leerwert 2 hatte $0,15 \mathrm{mod} / \mathrm{l} \mathrm{NaCl}$ als Probe,

Ansatz $B$ in Halbmikroküvetten (Plastibrand Cat. No. 759015):

$0,750 \mathrm{ml}$ Reagenzlösung,

$0,025 \mathrm{ml}$ Probe (Liquor, Plasma, Kontrollen),

$0,020 \mathrm{ml}$ Enzymsuspension (L-LDH plus GPT s. Ansatz A),

$0,020 \mathrm{ml} \mathrm{D}$-LDH-Suspension (s. Ansatz A).

Es wurden zwei Leerwerte entsprechend wie in Ansatz $A$ beschrieben ermittelt.

Die Messung erfolgte bei $334 \mathrm{~nm}$ im PCP 6121 mit Drucker (Eppendorf Gerätebau, Hamburg) nach 10,15 und 20 min bei Zimmertemperatur.

\section{Statistische Methoden}

Die Impräzision (intraseriell und interseriell) und Unrichtigkeit wurden nach (12) berechnet und die Nachweisgrenze als 3 Standardabweichungen des Leerwertes nach (12). Der Vergleich zweier Methoden $x$ und $y$ wurde nach (13) mit der Formel $y=b$ $+a x$ berechnet: Die Methoden $x$ und $y$ waren identisch bei $b=$ 0 und $a=1$ (13) 
Charakterisierung des modifizierten Monotests für D-Lactat und L-Lactat (Tab. 1)

Zur Messung von D- und L-Lactat in der gleichen Probe wurde der L-Lactat-Monotest von Boehringer Mannheim modifiziert und an Liquor in zwei Ansätze A und B adaptiert. Beide Modifikationen benötigen weniger Probe als bei $(8,9)$ und sind einfacher und schneller durchzuführen, da auf eine Enteiweißung der Probe mit Perchlorsäure verzichtet wurde.

Die Nachweisgrenze war bei jeweils 10 Messungen mit Leerwert 1 in beiden Ansätzen niedriger als mit Leerwert 2 (Tab. 1). Zur Messung von D-Lactat und L-Lactat im Ansatz $A$ und $B$ wurde deshalb der höhere Leerwert 2 verwendet und die Nachweisgrenze auf $0,20 \mathrm{mmol} / \mathrm{l}$ für D-Lactat und L-Lactat festgelegt.

Der Meßbereich wurde mit Kontrollen (Sigma) ermittelt, die gleiche Mengen an D- und L-Lactat enthielten. Ansatz $A$ ergab für beide Lactatenantiomere einen Meßbereich von $\leq 0,20$ bis 7,0 $\mathrm{mmol} / \mathrm{l}$, Ansatz $\mathrm{B} \leq 0,20$ bis $3,0 \mathrm{mmol} / \mathrm{l}$. Bei der alleinigen Messung von L-Lactat war, der Meßbereich größer (vgl. 2).

Tab. 1: Charakterisierung zweier Ansätze eines Lactat-Monotests zur enzymatischen Messung von D-Lactat und L-Lactat in der gleichen Probe.

\begin{tabular}{|c|c|c|c|}
\hline Parameter & & Ansatz A & Ansatz B \\
\hline Probenvolumen & & $\cdot 0,010 \mathrm{ml}$ & $0,025 \mathrm{ml}$ \\
\hline Gesamtvolumen & · & $0,330 \mathrm{ml}$ & $0,775 \mathrm{ml}$ \\
\hline Meßzeit & & $10 \mathrm{~min}$ & $20 \mathrm{~min}$ \\
\hline $\begin{array}{l}\text { Nachweisgrenzen } \\
\text { D-Lactat Leerwert } 1 \\
\text { D-Lactat Leerwert } 2 \\
\text { Nachweisgrenzen }\end{array}$ & & $\begin{array}{l}0,21 \mathrm{mmol} / \mathrm{I} \\
0,18 \mathrm{mmol} / \mathrm{l}\end{array}$ & $\begin{array}{l}0,09 \mathrm{mmol} / \mathrm{I} \\
0,22 \mathrm{mmol} / \mathrm{l}\end{array}$ \\
\hline $\begin{array}{l}\text { L-Lactat Leerwert } 1 \\
\text { L-Lactat Leerwert } 2\end{array}$ & & $\begin{array}{l}0,17 \mathrm{mmol} / 1 \\
0,20 \mathrm{mmol} / 1\end{array}$ & $\begin{array}{l}0,07 \mathrm{mmol} / \mathrm{I} \\
0,23 \mathrm{mmol} / \mathrm{l}\end{array}$ \\
\hline $\begin{array}{l}\text { Meßbereich } \\
\text { gleicher Mengen von } \\
\text { D-, L-Lactat im Ansatz }\end{array}$ & & $0-7 \cdot \mathrm{mmol} / \mathrm{l}$ & $0-3 \mathrm{mmol} / \mathrm{l}$ \\
\hline $\begin{array}{l}\text { Wiederauffindung von } \\
\text { D-Lactat } 0,44 \mathrm{mmol} / \mathrm{l} \\
\text { L-Lactat } 4,44 \mathrm{mmol} / \mathrm{l}\end{array}$ & . & $\begin{array}{l}89-112 \% \\
91-110 \%\end{array}$ & $\begin{array}{l}94-105 \% \\
96-103 \%\end{array}$ \\
\hline $\begin{array}{l}\text { Unrichtigkeit } \\
\text { D-Lactat } 2,19 \mathrm{mmol} / 1 \\
\text { L-Lactat } 4,44 \mathrm{mmol} / 1\end{array}$ & & $\begin{array}{l}4,5 \% \\
3,5 \%\end{array}$ & $\begin{array}{l}3,9 \% \\
3,1 \%\end{array}$ \\
\hline $\begin{array}{l}\text { Impräzision } \\
\text { D-Lactat } 2,19 \mathrm{mmol} / 1 \\
\text { intraseriell }(n=19) \\
\text { interseriell }(n=19) \\
\text { L-Lactat } 4,44 \mathrm{mmol} / 1\end{array}$ & & $\begin{array}{l}5,9 \% \text { VK } \\
7,7 \% \text { VK }\end{array}$ & $\begin{array}{l}4,7 \% \text { VK } \\
5,1 \% \text { VK }\end{array}$ \\
\hline $\begin{array}{l}\text { intraseriell }(n=20) \\
\text { interseriell }(n=20)\end{array}$ & $\cdots$ & $\begin{array}{l}4,1 \% \text { VK } \\
5,5 \% \text { VK }\end{array}$ & $\begin{array}{l}4,4 \% \text { VK } \\
5,0 \% \text { VK }\end{array}$ \\
\hline
\end{tabular}

Der Vergleich der Kontrollen $x$ ( $n=10$; mit $0,15 \mathrm{~mol} / 1 \mathrm{NaCl}$ verdünnt) mit den gemessenen Werten $y$ ergab keine signifikante Abweichung von $b=0$ und $a=1$ bei Berechnung mit der Gleichung $y=b+a x(13)$

Die Wiederfindung von $0,44 \mathrm{mmol} / \mathrm{D}$-Lactat in 9 Liquorproben streute stärker als diejenige von $4,44 \mathrm{mmol} / \mathrm{l}$ L-Lactat besonders im Ansatz $A$ (Tab. 1). Dies läßt sich auf eine hohe Impräzision der manuellen Lactatmessung besonders im Mikroansatz zurückführen (s. u.).

Die Impräzision der manuellen D-Lactatmessung zeigte interseriell größere Variationskoeffizienten (VK) als intraseriell besonders im Mikroansatz (Tab. 1). Die L-Lactat-Messung war etwas genauer (vgl. 2). Konzentrationen beider Laktatenantiomere unterhalb der Nachweisgrenze $(0,2$ bis $0 \mathrm{mmol} / \mathrm{l})$ konnten mit einer Impräzision zwischen 20 und $40 \%$ VK noch gemessen werden.

Um zu prüfen, in welcher Reihenfolge und Präzision geringe Mengen von D-Lactat zusammen mit großen Mengen von L-Lactat in der gleichen Probe gemessen werden können, wurden 58 Liquorproben mit 0 bis $0,8 \mathrm{mmol} / \mathrm{I} \mathrm{D}$-Lactat und 1,3 bis 16,2 $\mathrm{mmol} / \mathrm{l} \mathrm{L}$-Lactat wie folgt untersucht: Im Ansatz $A_{1}$ wurden zuerst L-Lactat und dann D-Lactat $(x)$, im Ansatz $A_{2} D$-Lactat und danach L-Lactat $(y)$ gemessen. D-Lactat- und L-Lactat-Konzentrationen $x$ und $y$ wurden nach (13) in der Gleichung $y=b+a x$ verglichen: Es zeigte sich, daß beide Ansätze identische Resultate lieferten (keine signifikante Abweichung von $b=0$ und $a=$ 1), jedoch war die Impräzision der D-Lactatmessung im Ansatz $A_{2}$ größer als im Ansatz $A_{1}$ (nicht dagegen für die relativ höheren L-Lactatkonzentrationen).

Daraus folgt, daß der oben beschriebene Ansatz $A_{1}$ für die $D$ Lactatmessung geeignet ist. Beide Ansätze $A$ und $B$ erscheinen zur Routine-Messung geringer Mengen von D-Lactat und höheren Konzentrationen von L-Lactat in der gleichen Probe ausreichend empfindlich und genau, da andere Techniken aufwendiger sind, wie z. B. die Kapillar-Gaschromatographie (14).

Aufstellung von Referenzbereichen für D-Lactat in Liquor und Blutplasma

In Lumballiquor- und Subokzipitalliquorproben von 25 Patienten mit Bandscheibenschaden und weitgehend im Referenzbereich $(3,15)$ liegenden Gehalten an Leukozyten, Gesamtprotein, Glucose und L-Lactat war D-Lactat nur bei 4 Patienten mit Werten von maximal $0,11 \mathrm{mmol} / \mathrm{l}$ nachweisbar; im gleichzeitig entnommenen Blutplasma wurden D-Lactatgehalte zwischen 0,1 und 0,2 $\mathrm{mmol} / \mathrm{l}$ gemessen (Tab. 2). (Ein meßbarer $\mathrm{D}$-Lactatgehalt im $\mathrm{Li}$ quor dürfte bei Kontrollen aus dem Blut stammen und unabhängig vom Entnahmeort im ZNS sein (vgl. 9)). In Anbetracht der hohen Impräzision in diesem Meßbereich (s. o.) wird die obere Grenze des Referenzbereichs für D-Lactat im Liquor cerebrospinalis und Blutplasma auf $0,20 \mathrm{mmol} / \mathrm{l}$ festgelegt. Dieser stimmt mit (8) überein, wo allerdings ein Grenzbereich zwischen 0,10 und $0,20 \mathrm{mmol} / /$ berücksichtigt würde. Der Referenzbereich für L-Lactat wurde im Lumballiquor von Erwachsenen auf 1,5 bis 2,1 $\mathrm{mmol} / \mathrm{l}$ festgesetzt $(3,15)$.

Tab. 2: D-Lactat und L-Lactat im Liquor cerebrospinalis bei Kontrollen und verschiedenärtigen Entzündungen des ZNS im Vergleich zu anderen Entzündungsparametern im Liquor

\begin{tabular}{|c|c|c|c|c|c|c|c|c|}
\hline Diagnose & $\begin{array}{l}\text { Alter } \\
\text { (Jahre) }\end{array}$ & $\begin{array}{l}\text { Liquor- } \\
\text { entnahme }\end{array}$ & $\begin{array}{l}\text { Leukozyten } \\
\text { pro } \mu \mathrm{l}\end{array}$ & $\begin{array}{l}\text { Erythrocyten } \\
\text { pro } \mu \mathrm{l}\end{array}$ & $\begin{array}{l}\text { Gesamt } \\
\text { protein } \\
\text { g/l }\end{array}$ & $\begin{array}{l}\text { Glucose } \\
\text { mg/dl }\end{array}$ & $\begin{array}{l}\text { L-Lactat } \\
\mathrm{mmol} / \mathrm{I}\end{array}$ & $\begin{array}{l}\text { D-Lactat } \\
\text { mmol// }\end{array}$ \\
\hline $\begin{array}{l}\text { Bandscheiben- } \\
\text { schaden (15) }\end{array}$ & $\begin{array}{l}52^{*} \\
26-75\end{array}$ & lumbal & $\begin{array}{l}2^{*} \\
1-4\end{array}$ & $\begin{array}{l}5^{*} \\
0-99\end{array}$ & $\begin{array}{l}0,35^{*} \\
0,25-0,45\end{array}$ & $\begin{array}{l}61^{*} \\
50-85\end{array}$ & $\begin{array}{l}1,4^{*} \\
0,9-2,3\end{array}$ & $\begin{array}{l}0^{* *} \\
0^{* *}-0,11^{*}\end{array}$ \\
\hline $\begin{array}{l}\text { Bandscheiben- } \\
\text { schaden (10) }\end{array}$ & $\begin{array}{l}49 \\
30-70\end{array}$ & SOP & $\begin{array}{l}1 \\
1-3\end{array}$ & $\begin{array}{l}5 \\
0-198\end{array}$ & $\begin{array}{l}0,27 \\
0,14-0,37\end{array}$ & $\begin{array}{l}56 \\
49-70\end{array}$ & $\begin{array}{l}1,3 \\
0,2-1,6\end{array}$ & $\begin{array}{l}0^{* *} \\
0^{* *}-0,10\end{array}$ \\
\hline $\begin{array}{l}\text { Meningitis (23) } \\
\text { bakteriell akut }\end{array}$ & $\begin{array}{l}50 \\
5-63\end{array}$ & $\begin{array}{l}\text { Ventrikel } \\
\text { lumbal }\end{array}$ & $\begin{array}{l}204 \\
5-21504\end{array}$ & $\begin{array}{l}409 \\
13-54682\end{array}$ & $\begin{array}{l}1,46 \\
0,15-10,1\end{array}$ & $\begin{array}{l}51 \\
0-92\end{array}$ & $\begin{array}{l}7,8 \\
2,3-17,8\end{array}$ & $\begin{array}{l}0,49 \\
0,24-1,28\end{array}$ \\
\hline $\begin{array}{l}\text { Meningitis ( } 51 \text { ) } \\
\text { bakteriell } \\
\text { anbehandelt }\end{array}$ & $\begin{array}{l}54 \\
1-63\end{array}$ & $\begin{array}{l}\text { Ventrikel } \\
\text { lumbal }\end{array}$ & $\begin{array}{l}115 \\
2-46080\end{array}$ & $\begin{array}{l}259 \\
0-3110000\end{array}$ & $\begin{array}{l}0,93 \\
0,1-5,3\end{array}$ & $\begin{array}{l}55 \\
0-157\end{array}$ & $\begin{array}{l}4,3 \\
1,5-17,7\end{array}$ & $\begin{array}{l}0,04 \\
0^{* *}-0,45\end{array}$ \\
\hline $\begin{array}{l}\text { Meningitis (17) } \\
\text { Verdacht }\end{array}$ & $\begin{array}{l}39 \\
1-73\end{array}$ & $\begin{array}{l}\text { lumbal } \\
\text { Ventrikel }\end{array}$ & $\begin{array}{l}67 \\
6-518\end{array}$ & $\begin{array}{l}22 \\
0-1600\end{array}$ & $\begin{array}{l}0,62 \\
0,28-1,79\end{array}$ & $\begin{array}{l}60 \\
40-90\end{array}$ & $\begin{array}{l}2,8 \\
1,4-5,4\end{array}$ & $\begin{array}{l}0,12 \\
0^{* *}-2,13\end{array}$ \\
\hline $\begin{array}{l}\text { Blutungen (60) } \\
\text { in die Liquorräume }\end{array}$ & $\begin{array}{l}49 \\
11-70\end{array}$ & Ventrikel & $\begin{array}{l}31 \\
1-122880\end{array}$ & $\begin{array}{l}8243 \\
237-408112\end{array}$ & $\begin{array}{l}1,37 \\
0,13-13,00\end{array}$ & $\begin{array}{l}90 \\
35-156\end{array}$ & $\begin{array}{l}4,2 \\
1,2-7,8\end{array}$ & $\begin{array}{l}0,10 \\
0 * *-0,31\end{array}$ \\
\hline
\end{tabular}

- Median mit 0 und 100 Perzentil

- In Ansat? A und B nicht nactiweisbar 
D-Lactat und L-Lactat im Liquor cerebrospinalis bei verschiedenen Entzündungen im ZNS

In 23 akuten bakteriellen Meningitiden wurden D-Lactatwerte zwischen 0,24 und $1,28 \mathrm{mmol} / \mathrm{l}$ im Ventrikel-oder Lumballiquor gemessen mit L-Lactatwerten zwischen 2,3 und $17,8 \mathrm{mmol} / \mathrm{I}$ (Tab. 1). Im simultan entnommenen Blutplasma waren die $D$ Lactatwerte stets niedriger als im Liquor. Über vergleichbar hohe D-Lactatgehalte wurde im Liquor bei bakteriellen Meningitiden von (9) berichtet. Gleichzeitig mit den erhöhten Lactatwerten wurden vermehrte Gehalte an Leukozyten und Gesamtprotein im Liquor beobachtet (mit oder ohne Erythrozyten), was die akute bakterielle Entzündung im ZNS untermauert. Der Glucosegehalt war weniger häufig vermindert (Tab. 1). Bei einigen Proben von Ventrikeldrainageliquor wurden erhöhte D-Lactatwerte bei nur leicht vermehrten Gehalten an Leukozyten und Gesamtprotein gefunden (Tab. 1), was auf eine externe Kontamination mit Bakterien hinweist. Allerdings war der D-Lactatgehalt im Liquor von zwei Patienten mit eindeutigem Bakteriennachweis $<0,20 \mathrm{mmol} / /$ bei stark erhöhten Werten von L-Lactat, Gesamtprotein und der Leukozytenzahl. Hier dürfte es sich um nicht-DLactat-produzierende Bakterien handeln!

Nach Anbehandlung mit Antibiotika wurden bei 4 von 51 bakteriellen Meningitiden $D$-Lactatwerte von $>0,20 \mathrm{mmol} / \mathrm{l}$ gemessen bei einem L-Lactatgehalt von $>3,5 \mathrm{mmol} / /$ (Tab. 1). Bei den restlichen 47 Patienten mit nicht erhöhtem D-Lactatgehalt wurden häufig erhöhte Werte von L-Lactat, Gesamtprotein und Leukozyten sowie erniedrigte Glucosewerte gefunden (Tab. 1). Damit kann mit Hilfe der Lactatdifferenzierung zwischen Bakterien-spezifischem D-Lactat und Entzündungsreaktionen wie L-Lactatazidose im Liquor und Blut unterschieden und die Wirkung der Antibiotikatherapie kontrolliert werden.

Bei 17 Patienten mit dem klinischen Verdacht einer akuten Meningitis und erhöhten Leukozytenzahlen im Liquor wurde nur in zwei Fällen ein D-Lactatgehalt von $>0,20 \mathrm{mmol} / \mathrm{l}$ beobachtet, wobei ein Patient einen L-Lactatwert von $>3,5 \mathrm{mmol} / \mathrm{l}$ aufwies. Damit kann mit Hilfe der Lactatdifferenzierung zwischen abakterieller und bakterieller Meningitis offensichtlich besser differenziert werden als mit dem L-Lactat-Test allein.

Bei 60 Blutungen in die Liquorräume war der D-Lactatgehalt im drainierten Ventrikelliquor bei 7 Patienten $>0,20 \mathrm{mmol} / \mathrm{l}$, außerdem waren Leukozytenzahlen, L-Lactat und Gesamtprotein erhöht. Der Glucosewert war nur selten erniedrigt (Tab. 1). Diese Befunde indizieren eine akute bakterielle Entzündung in den blutigen Liquorproben!

Bei einigen dieser Intensiv-Pflege-Patienten wurden im Blutplasma D-Lactatwerte von $>0,20 \mathrm{mmol} / \mathrm{l}$ gefunden. Die Liquorwerte lagen zwischen 0 und $\geq 0,20 \mathrm{mmol} / / \mathrm{D}$-Lactat. Da D-Lactat auch bei extrazerebralen Entzündungen (8) und bei Darmerkrankungen im Blut ansteigt (vgl. 16-19) und damit zu meßbaren Werten in Liquor führt, muß D-Lactat bei allen unklaren akuten Entzündungsvorgängen simultan in Liquor und Blut gemessen werden, um den Entzündungsprozeß sicher zu lokalisieren.

Zusammenfassend wird festgestellt, daß die einfache und schnelle Messung von D-Lactat und L-Lactat in simultan gewonnenen Liquor- und Blutproben mit Hilfe eines hier modifizierten Monotests eine Bereicherung in der Notfalldiagnostik von akuten Entzündungen darstellt.
Schriftum:

1. KLeine, T. O., Baenlocher, K., Niederer, V., Keller, H., ReUtTer, F., TRITSCHLER, W. BABLOK, W: Diagnostische Bedeutung der Lactatbestimmung im Liquor bel Meningitis. Dtech. med. Wachr. 104, 553-557 (1979).

2. KLEINE, T. O.: Neue Labormethoden für die Liquordiagnostik. Thieme Verlag Stutt-

gart (1980).

3. KLEINE, T. O.: Nervensystem, In: GREILING, H., GRESSNER, A. M. (Hrsg.): Lehrbuch der Klinischen Chemis und Pathobiochemie. Schattauer Verlag, 859-893 (1989).

4. BLAND, R. D., LISTER, R. C.. RIES, J. P.: Cerobrospinal fluld lactic acld lovel and pH in moningitis. Alds in differential diagnosis. Am. J. Dis. Child 128, 151 - 156 (1974). 6. CONTRONI, G., RODRIGUEZ, W. J., HICKS, J. M., FICKE, M., ROSS, S., FRIEDMAN, G., KHAN, W.: Cerebrospinal fluid loctic acid levels in meningitis. J. Pediatr. 9, 379-384 (1977).

6. BROOK, I., BRICKNELL, K. S., OVERTURF, G. D., FINEGOLD, S. M.: Measurement of lactic acid in cerebrospinal fluid of patients with infections of the central nervous systom. J. Infoct. Dis. 137, 384-390 (1978).

7. KLEINE, T. O., SCHLENSKA, G. K.: Evaluation of three polymorphonuclear leukocyte constituents in CSF and comparison with lactate content and leukocyte counts for the constituents in CSF and comparison with lactate content and leukocyte counts

8. SMITH, S. M., ENG, R. H. K., CAMPOS, J. M., CHMEL, H.: D-Lactic acid measurements in the diagnosis of bacterial infections. J. Clin. Microbiol. 27, 385-388 (1989). 9. SMITH, S. M., ENG, R. H. K., BUCCINI, F.: Use of D-lactic acid measurements in the diagnosis of bacterial infections. J. Infect. Dis. 154, 658-664 (1986).

10. NOLL, F.: L-Lactat, Bestimmung mit LOH, GPT und NAD. In: BERGMEYER, H. U. (Hrsg.): Methoden der enzymatischen Analyse. 3. Aufl., Bd. 2, 1521 - 1525. Verlag Chemle, Weinheim.

11. GAWEHN, K., BERGMEYER, H. U.: D-(-)-Lactat. In: BERGMEYER, H. U. (Hrsg.): Methoden der enzymatischen Analyse. 3. Aufl. Bd. 2, 1538-1541. Verlag Chemie, Weinheim.

12. STAMM, D.: Der analytische Teilschritt und seine Zuverlässigkeit. In: GREILING, H. GRESSNER, A. M. (Hrsg.): Lehrbuch der Klinischen Chemie und Pathobiochemie. Schattauer Verlag, Stuttgart, 8-30 (1989).

13. PASSING, H., BABLOK, W.: A new biometrical procedure for testing the equality of measurements from different analytical methods. J. Clin. Chem. Clin. Biochem. 21, 709-720 (1983).

14. KAMERLING, J. P., GERWIG, G. J., VLIEGENTHART, F. G., DURAN, M., KETTING, D. WADMAN, S. K.: Determination of the configuration of lactic and glyceric acids from human serum and urine by capillary gas-liquid chromatography. J. Chromatography, 117-123 (1977).

15. KLEINE, T. O.: Liquor. In: THOMAS, L. (Hrsg.): Labor und Diagnose. Medizinische 15. KLEINE, T. O.: Liquor. In: THOMAS, L. (H

16. SCHOOREL, E. P., GIESBERTS, M. A. H., BLOM, W., VAN GELDEREN, H. H.: D-Lactic 16. SCHOOREL, E. P., GIESBERTS, M. A. H., BLOM, W., VAN GELDEREN, H. H.: D-Lact
acidosis in a boy with short bowel syndrome. Arch. Dis. Child 55, 810-812 (1980). 17. CARR, D. B., SHIH, V. E., RICHTER, J. M., MARTIN, J. B.: D-Lactic acidosis simuitating a hypothalanic syndrome after bowel bypass. Ann. Neurol. 11, 195- 197 (1982). 18. STOLBERG, L., ROLFE, R., GITLIN, N., MERRI, J., MANN, L., LINDER, J., FINEGOLD, S.: D-Lactic acidosis due to abnormal gut flora. Diagnosis and treatment of two cases. N. Engl. J. Med. 306, 1344-1348 (1982).

19. OH, M. S., PHELPS, K. R., TRAUBE, M., BARBOSA-SALDIVAR, J. L, BOXHILL, C., CARROLL, H. J.: D-Lactic acidosis in a man with the short-bowel syndrome. N. Engl. J. Med. 301, 249-252 (1979).

\section{Danksagung}

Frau Petra Nebel danke ich für ihre zuverlässige Hilfe bei den Lactatmessungen. Diese Untersuchungen wurden finanziell durch den Bundesminister für Forschung und Technologie gefördert (Förderungszeichen 01 ZR 8513).

\section{Anschrift des Verfassers:}

Prof. Dr. T. O. Kleine

Med. Zentrum für Nervenheilkunde

Funktionsbereich Neurochemie

Rudolf-Bultmann-Str. 8

3550 Marburg

-

\section{Sponsoren und ausstellende Firmen*:}

Becton Dickinson GmbH, Heidelberg* Behringwerke AG, Marburg und Frankfurt* Digitana AG, Frankfurt

A. Hettich Zentrifugen, Tuttlingen* Hoffmann-La Roche, Grenzach-Wyhlen E. Merck, Darmstadt*
Nordmark Arzneimittel GmbH, Uetersen

Pharmacia LKB GmbH, Freiburg*

Sarstedt, Nümbrecht

Fa. Syva Diagnostica, Darmstadt*

Universität Marburg 